C-G LIU

KODAI MATH. J.

9 (1986), 368-384

\title{
NECESSARY AND SUFFICIENT CONDITIONS FOR A POISSON APPROXIMATION (TRIVARIATE CASE)
}

\author{
By Cheng-GeE LIU
}

\section{Introduction.}

In paper [1], M. Polak has shown that V. R. Mises (1921) has derived sufficient conditions of Poisson approximation for sums of independent univariate Bernoulli random variables which may not be identically distributed, and that J. Macys (1977) has derived that the converse assertion is true, i.e. the conditions are necessary for Poisson approximation as well. M. Polak (1982) has extended the univariate case to bivariate case. In this paper, we want to extend Polak's results [1], and generalize Kawamura's results [2] to trivariate case.

Before showing the main results, we give the following notations and definitions.

\section{Notations and definitions.}

$g, k, m, n:$ positive integers,

$\left\{\boldsymbol{e}_{1}=(1,0,0), \boldsymbol{e}_{2}=(0,1,0), \boldsymbol{e}_{3}=(0,0,1)\right\}$ : base of 3 dimensional vectors,

$E=\left\{e_{1}, e_{2}, e_{3}, e_{1}+e_{2}, e_{1}+e_{3}, e_{2}+e_{3}, e_{1}+e_{2}+e_{3}\right\}$,

$\boldsymbol{i}$ : 3 dimensional vector belonging to $\boldsymbol{E}$,

$\boldsymbol{s}=\left(s_{1}, s_{2}, s_{3}\right): 3$ dimensional vector,

$\left(\begin{array}{l}n \\ m\end{array}\right)=n ! /[m !(n-m) !]$,

$A_{i}$ : frequence of the observation $i$ in $n_{k}$ trivariate Bernoulli trials,

$t_{j}^{i}$ : the trial number for the $j$-th occurrence of observation $i$ in the $n_{k}$ trials with $t_{j}^{\boldsymbol{i}} \in\left\{1, \cdots, n_{k}\right\}$, where $j=1,2, \cdots, A_{\imath}$,

$F_{\dot{i}}=\left\{\left(t_{1}^{i}, t_{2}^{i}, \cdots, t_{A_{i}}^{i}\right) ; t_{1}^{i}<t_{2}^{i}<\cdots<t_{A_{i}}^{i}\right\}$,

$G_{\dot{\boldsymbol{i}}}$ : the set of integers expressed in $\left(t_{1}^{t}, \cdots, t_{A_{\boldsymbol{i}}}^{\boldsymbol{t}}\right)$ belonging to $F_{\boldsymbol{t}}$ denoted as $G_{i}=\left\{t_{1}^{i}, \cdots, t_{A_{i}}^{i}\right\}$,

$\sum_{F_{\dot{i}}}:$ the sum of all terms for $\left(t_{1}^{i}, \cdots, t_{A_{\dot{\boldsymbol{i}}}}^{i}\right) \in F_{a}$,

$\sum_{G_{i n}\left(G_{e_{1}} \cup G_{e_{2}} \cup \cdots\right)=\varnothing}$ : the sum of all terms for $\left(t_{1}^{i}, \cdots, t_{A_{i}}^{\boldsymbol{t}}\right) \in F_{t}$, with the condition $G_{i} \cap\left\{G_{e_{1}} \cup G_{e_{2}} \cup \cdots\right\}=\varnothing$,

Received December 17, 1985 
$\boldsymbol{A}=\left(A_{e_{1}}, A_{e_{2}}, A_{e_{3}}, A_{e_{1}+e_{2}}, A_{e_{1}+e_{3}}, A_{e_{2}+e_{3}}, A_{e_{1}+e_{2}+e_{3}}\right)$, $[C]=\left[\boldsymbol{A} ; \sum_{\left\langle i, e_{j}=1\right.} A_{\boldsymbol{i}}=s_{\jmath}, j=1,2,3\right]$, where we can obtain $A_{\imath} \leqq \max _{\jmath} s$, for every $\boldsymbol{i}$, $\sum_{[C]}$ : the sum of all terms for $A_{\imath}$ 's with the restriction of $[C]$,

$\lambda_{i}$ : nonnegative real parameter for every $i \in E$,

$t_{r_{n}}^{i}, t_{s_{n}}^{i}$ : integers which are consisting with the elements of $G_{i}$ with $r_{n}, s_{n} \in$ $\left\{1,2, \cdots, A_{\boldsymbol{i}}\right\}$, where $n$ is positive integer.

$A_{n_{k}}(\boldsymbol{A}), B_{n_{k}}(\boldsymbol{A}), C_{n_{k}}(\boldsymbol{A})$ : sum of the product of probabilities which will be deduced later from (2.2.1), (2.8) and (2.1.1).

\section{Conditions sufficient for Poisson approximation.}

Let $\left\{\boldsymbol{X}_{k j}=\left(X 1_{k \jmath}, X 2_{k \jmath}, X 3_{k j}\right), j=1,2, \cdots, n_{k}\right\}$ be a sequence of independent trivariate Bernoulli vectors for every $k \geqq 1$ with

$$
P\left[\boldsymbol{X}_{k j}=\boldsymbol{i}\right]=P_{k j}(\boldsymbol{i}), \quad \text { for every } \boldsymbol{i} \in \boldsymbol{E} \cup\{0\},
$$

where

$$
\sum_{i \in E \cup(0\}} P_{k j}(i)=1
$$

To explain $\boldsymbol{X}_{k j}\left(j=1,2, \cdots, n_{k}\right)$, we may consider the following example for $n_{k}=16$.

\section{Example 1.}

$$
\begin{array}{llllllllllllllllll}
j & 1 & 2 & 3 & 4 & 5 & 6 & 7 & 8 & 9 & 10 & 11 & 12 & 13 & 14 & 15 & 16 & \text { sum } \\
X 1_{k \jmath} & 0 & 1 & 0 & 1 & 0 & 0 & 1 & 1 & 1 & 0 & 1 & 0 & 1 & 0 & 1 & 1 & 9 \\
X 2_{k \jmath} & 0 & 1 & 1 & 0 & 0 & 0 & 0 & 1 & 0 & 1 & 0 & 1 & 0 & 0 & 0 & 0 & 5 \\
X 3_{k \jmath} & 1 & 0 & 1 & 1 & 0 & 1 & 0 & 1 & 0 & 0 & 0 & 0 & 0 & 1 & 1 & 0 & 7
\end{array}
$$

Let us denote $\boldsymbol{S}_{k}=\sum_{j=1}^{n_{k}} \boldsymbol{X}_{k j}=\sum_{j=1}^{n_{k}}\left(X 1_{k \jmath}, X 2_{k \jmath}, X 3_{k j}\right)$ for every $k \geqq 1$. In this example, we have $\boldsymbol{S}_{k}=(9,5,7)$. However, in the following discussion $P_{k j}(\boldsymbol{i})$ expressed in (2.0) will be replaced by $P_{j}(\boldsymbol{i})$ for simplicity. Then $P\left[\boldsymbol{S}_{k}=\boldsymbol{s}\right]$ can be expressed easily as follows.

$$
\begin{aligned}
& P\left[\boldsymbol{S}_{k}=\boldsymbol{s}\right]=\sum_{[C]}\left\{\sum_{\boldsymbol{F}_{\boldsymbol{e}_{1}}}\left[\prod_{j=1}^{\boldsymbol{A}_{e_{1}}} P_{t_{j}^{e_{1}}}\left(\boldsymbol{e}_{1}\right)\right]\right.
\end{aligned}
$$

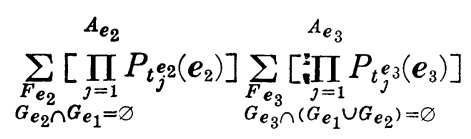

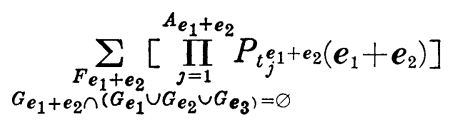




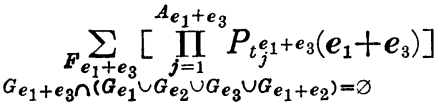

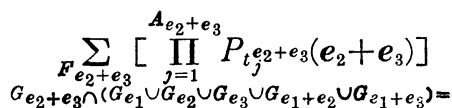

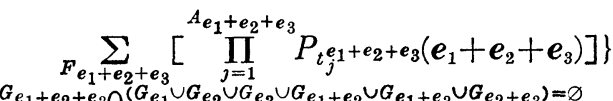

$$
\begin{aligned}
& \prod_{g=1}^{n_{k}} P_{\boldsymbol{g}}(\mathbf{0}) \\
& g \notin\left(G_{e_{1}}^{g=1} \cup G_{e_{2}} \cup G_{e_{3}} \cup G_{e_{1}+e_{2}} \cup G_{e_{1}+e_{3}} \cup G_{e_{2}+e_{3}} \cup G_{e_{1}+e_{2}+e_{3}}\right)
\end{aligned}
$$

For simplicity, if the term in the braces $\{\cdots\}$ of $(2.1)$ is replaced by $C_{n_{k}}(\boldsymbol{A})$, then we have

$$
P\left[\boldsymbol{S}_{k}=\boldsymbol{s}\right]=\sum_{[C]}\left\{C_{n_{k}}(\boldsymbol{A})\right\} \prod_{\substack{g=1 \\ g \in\left(G_{e_{1}} \cup G_{e_{2}} \cup G_{e_{3}} \cup G_{e_{1}+e_{2}} \cup G_{e_{1}+e_{3}} \cup G_{e_{2}}+e_{3} \cup G_{e_{1}}+e_{2}+e_{3}\right)}}^{n_{k}} P_{g}(\mathbf{0})
$$

and also by (2.1) we have

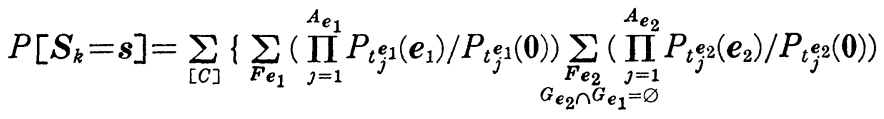

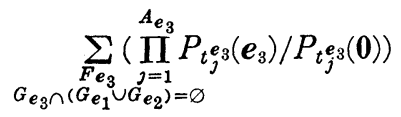

$$
\begin{aligned}
& \sum_{F_{e_{1}+e_{2}}}\left(\prod_{j=1}^{A_{e_{1}+e_{2}}} P_{t_{j}^{e_{1}+e_{2}}}\left(\boldsymbol{e}_{1}+\boldsymbol{e}_{2}\right) / P_{t_{j}^{e_{1}+e_{2}}}(\mathbf{0})\right) \\
& \sum_{F_{e_{1}+e_{3}}}\left(\prod_{\substack{j=1 \\
G_{e_{1}}+e_{3} \cap\left(G_{e_{1}} \cup G_{e_{2}} \cup G_{e_{3}} \cup G_{e_{1}}+e_{2}\right)=\varnothing}}^{A_{e_{1}+e_{3}}} P_{e_{1}^{e_{1}+e_{3}}}\left(\boldsymbol{e}_{1}+\boldsymbol{e}_{3}\right) / P_{t_{j}^{e_{1}}+e_{3}}(0)\right) \\
& \sum_{F_{e_{2}+e_{3}}}\left(\prod_{j=1}^{A_{e_{2}+e_{3}}} P_{t_{j}^{e_{2}+e_{3}}}\left(\boldsymbol{e}_{2}+\boldsymbol{e}_{3}\right) / P_{t_{j}^{e_{2}+e_{3}}}(0)\right)
\end{aligned}
$$

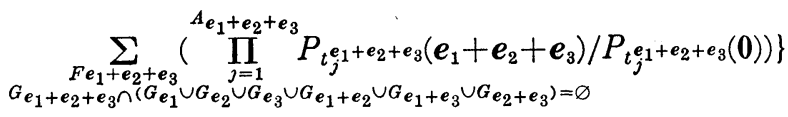

$$
\begin{aligned}
& \prod_{g=1}^{n_{k}} P_{g}(\mathbf{0}) .
\end{aligned}
$$

Similarly, if the term in the braces $\{\cdots\}$ of $(2.2)$ is replaced by $A_{n_{k}}(\boldsymbol{A})$, then we have 


$$
P\left[\boldsymbol{S}_{k}=\boldsymbol{s}\right]=\sum_{[C]} \prod_{g=1}^{n_{k}}\left\{A_{n_{k}}(\boldsymbol{A})\right\} P_{\boldsymbol{g}}(\mathbf{0})
$$

THEOREM 1. If the following conditions (2.3) and (2.4) are satisfied for the sequence of independent Bernoulli distribution which may not be identically distributed,

$$
\begin{aligned}
& \sum_{j=1}^{n_{k}} P_{k j}(\boldsymbol{i}) \rightarrow \lambda_{2} \quad \text { as } \quad k \rightarrow \infty \quad \text { for all } \boldsymbol{i} \in \boldsymbol{E}, \\
& \min _{1 \leqq j \leqq n_{k}} P_{k j}(\mathbf{0}) \rightarrow 1 \quad \text { as } \quad k \rightarrow \infty,
\end{aligned}
$$

then we have

$$
\lim _{k \rightarrow \infty} P\left[\boldsymbol{S}_{k}=\boldsymbol{s}\right]=\sum_{[C]} \frac{\lambda_{e_{1}}^{A_{e_{1}}} \lambda_{e_{2}}^{A} A_{e_{2}} \cdots \lambda_{e_{1}+e_{2}+e_{3}}^{A_{e_{1}+e_{2}+e_{3}}}}{A_{e_{1}} ! A_{e_{2}} \cdots ! A_{e_{1}+e_{2}+e_{3}} !} e^{-\left(\lambda_{e_{1}}+\lambda_{e_{2}}+\cdots+\lambda_{e_{1}+e_{2}+e_{3}}\right)}
$$

for every s, where $[C]=\left[A ; \sum_{\left\langle i \cdot e_{j}\right\rangle=1} A_{i}=s_{\jmath}, j=1,2,3\right]$.

Proof. In order to prove the theorem, we consider the following three steps.

(step 1) We want to prove that

$$
\prod_{g=1}^{n_{k}} P_{g}(\mathbf{0}) \rightarrow e^{-\left(\lambda_{e_{1}}+\lambda_{e_{2}}+\cdots+\lambda_{\left.e_{1}+e_{2}+e_{3}\right)}\right.} \quad \text { as } \quad k \rightarrow \infty .
$$

Consider the inequality

$$
1+y \leqq e^{y}, \quad y \in[-1, \infty),
$$

putting $y=-x$ and $y=x /(1-x), x \in[0,1)$, we obtain $e^{-x /(1-x)} \leqq 1-x \leqq e^{-x}$, $x \in[0,1)$. Now putting $\Delta_{g}=P_{g}\left(\boldsymbol{e}_{1}\right)+P_{g}\left(\boldsymbol{e}_{2}\right)+\cdots+P_{g}\left(\boldsymbol{e}_{1}+\boldsymbol{e}_{2}+\boldsymbol{e}_{3}\right)=1-P_{\boldsymbol{g}}(\boldsymbol{0})$, where $0 \leqq \Delta_{g}<1$ (by (2.4)) for sufficiently large $k\left(1 \leqq g \leqq n_{k}\right)$, and using the last inequality, we get

$$
e^{-\frac{1}{\min P} \bar{g}^{(0)}} \stackrel{\sum}{g=1}_{\sum_{k}}^{n_{k}} L_{g} \leqq \prod_{g=1}^{n_{k}} P_{g}(0) \leqq e^{-} \sum_{g=1}^{n_{k}} \Delta_{g},
$$

and from (2.3), (2.4) we can prove that

$$
\prod_{\jmath=1}^{n_{k}} P_{g}(\mathbf{0}) \rightarrow e^{-\left(\lambda e_{1}+\lambda_{e_{2}}+\cdots+\lambda_{e_{1}}+e_{2}+e_{3}\right)} \quad \text { as } \quad k \rightarrow \infty .
$$

(step 2) In order to derive the limiting value of (2.1), we need to prove (2.8) by (2.7). In this step, let us prove (2.7) and (2.8). Let us put

$$
B_{n_{k}}(\boldsymbol{A})=\sum_{F_{e_{1}}} \prod_{j=1}^{A_{e_{1}}} P_{t_{j}} e_{1}\left(\boldsymbol{e}_{1}\right) \sum_{F_{e_{2}}} \prod_{j=1}^{A_{e_{2}}} P_{t_{j} e_{2}}\left(\boldsymbol{e}_{2}\right) \sum_{F_{e_{3}}} \prod_{j=1}^{A_{\boldsymbol{e}_{3}}} P_{t_{j}} \boldsymbol{e}_{3}\left(\boldsymbol{e}_{3}\right)
$$




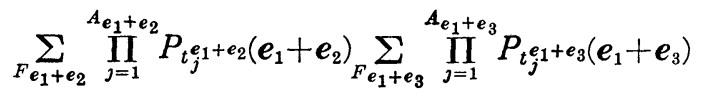

$$
\begin{aligned}
& \sum_{F_{2}+e_{3}} \prod_{j=1}^{A_{e_{2}+e_{3}}} P_{t_{j}^{e_{2}}+e_{3}}\left(e_{2}+e_{3}\right)
\end{aligned}
$$

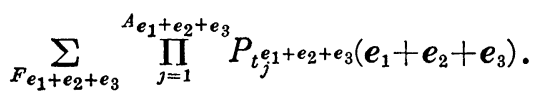

Now we are going to prove that

$$
\sum_{F_{i}}\left[\prod_{j=1}^{A i} P_{t_{j}^{i}}(i)\right] \rightarrow \lambda_{e}^{A i} / A_{\imath} ! \quad \text { for every } \quad \boldsymbol{i} \in \boldsymbol{E} .
$$

The proof is given by induction with respect to $A_{\mathbf{x}}$.

(1) $A_{\boldsymbol{i}}=1$. By (2.3), it is obvious that

$$
\sum_{t_{1}^{i}=1}^{n_{k}} P_{t_{1}^{i}}(i) \rightarrow \lambda_{i} \quad \text { as } \quad k \rightarrow \infty .
$$

(2) $A_{i}=2$. By (2.3) and (2.4), we have

because

$$
\sum_{t_{1}^{i}<t_{2}^{i}} P_{t_{1}^{i}}(\boldsymbol{i}) P_{t_{2}^{i}}(\boldsymbol{i}) \rightarrow \lambda_{d}^{2} / 2,
$$

$$
0 \leqq \sum_{g=1}^{n_{k}} P_{g}^{2}(\boldsymbol{i}) \leqq\left(1-\min _{\boldsymbol{g}} P_{g}(\mathbf{0})\right) \sum_{g=1}^{n_{k}} P_{g}(\boldsymbol{i}),
$$

and by (2.3), (2.4) the right hand side of the inequality tends to 0 , so we have

$$
2 \sum_{t_{1}^{i}<t_{2}^{i}} P_{t_{1}^{i}}(\boldsymbol{i}) P_{t_{2}^{i}}(\boldsymbol{i})=\left[\sum_{\boldsymbol{g}=1}^{n_{k}} P_{\boldsymbol{g}}(\boldsymbol{i})\right]^{2}-\sum_{\boldsymbol{g}=1}^{n_{k}} P_{\boldsymbol{g}}^{2}(\boldsymbol{i}) \rightarrow \lambda_{\boldsymbol{\imath}}^{2} \quad \text { as } \quad k \rightarrow \infty .
$$

(3) Assume that (2.7) is correct as $A_{i}=m-1$, that is,

$$
\sum_{t_{1}^{\boldsymbol{i}}<\cdots<t_{m-1}^{\boldsymbol{i}}} \prod_{j=1}^{m-1} P_{t_{j}^{i}}(\boldsymbol{i}) \rightarrow\left(\lambda_{\boldsymbol{i}}\right)^{m-1} /(m-1) ! \quad \text { as } \quad k \rightarrow \infty .
$$

In order to finish tne induction, let us prove (2.7) as $A_{i}=m$.

Multiply the left hand side of the last relation by $\sum_{t_{m}^{i}=1}^{n_{k}} P_{t_{m}^{i}}(i)$ which tends to $\lambda_{i}$ (by (2.3)), we obtain

$$
\begin{aligned}
& \quad \sum_{t_{1}^{i}<\cdots<t_{m-1}^{i}} P_{t_{1}^{i}}(\boldsymbol{i}) \prod_{j=1}^{m-1} P_{t_{j}^{i}}(\boldsymbol{i})+\sum_{t_{1}^{i}<\cdots<t_{m-1}^{i}} P_{t_{2}^{i}}(\boldsymbol{i}) \prod_{j=1}^{m-1} P_{t_{j}^{i}}(\boldsymbol{i})+\cdots \\
& +\sum_{t_{1}^{i}<\cdots<t_{m-1}^{i}} P_{t_{m-1}^{i}}(\boldsymbol{i}) \prod_{j=1}^{m-1} P_{t_{j}^{i}}(\boldsymbol{i})+\sum_{t_{m}^{i}<t_{1}^{i}<\cdots<t_{m-1}^{i}} \prod_{j=1}^{m} P_{t_{j}^{i}}(\boldsymbol{i}) \\
& +\sum_{t_{1}^{i}<t_{m}^{i}<t_{2}^{i}<\cdots<t_{m-1}^{i}} \prod_{j=1}^{m} P_{t_{j}^{i}}(i)+\cdots+\sum_{t_{1}^{i}<\cdots<t_{m-1}^{i}<t_{m}^{z}} \prod_{j=1}^{m} P_{t_{j}^{i}}(\boldsymbol{i}) .
\end{aligned}
$$


Each the first $(m-1)$ terms of (2.7.1) may be nonnegative and estimated by

$$
\left[1-\min _{g} P_{g}(0)\right]_{t_{1}^{i}<\cdots<t_{m-1}^{i}} \prod_{j=1}^{m-1} P_{t_{j}^{i}}(i)
$$

which is an upper bound of these terms and tends to 0 ; that is,

$$
\begin{aligned}
& 0 \leqq[\text { each of the first }(m-1) \text { terms of (2.7.1)] } \\
& \leqq\left[1-\min _{\boldsymbol{g}} P_{\boldsymbol{g}}(\mathbf{0})\right] \sum_{t_{\mathbf{1}}^{i}<\cdots<t_{m-1}^{i}} \prod_{j=1}^{m-1} P_{t_{j}^{i}}(\boldsymbol{i}) .
\end{aligned}
$$

So each of the first $(m-1)$ terms tends to 0 , and each of the last $m$ terms has the same value, then we can obtain the limiting value of (2.7.1) to be

$$
m \sum_{t_{1}^{i}<\cdots<t_{m}^{i}} \prod_{j=1}^{m} P_{t_{j}^{i}}(\boldsymbol{i}) \rightarrow \lambda_{i} \cdot\left(\lambda_{\boldsymbol{i}}\right)^{m-1} /(m-1) ! ;
$$

that is, (2.7) is correct as $A \boldsymbol{i}=m$ and we finish the proof of (2.7) by the induction. Then by (2.7), we have

$$
B_{n_{k}}(\boldsymbol{A}) \rightarrow \frac{\lambda_{e_{1}}^{A_{e_{1}} \lambda_{e_{2}}^{A_{e_{2}}} \cdots \lambda_{e_{1}+e_{2}+e_{3}}^{A_{e_{1}}+e_{2}+e_{3}}}}{A_{e_{1}} ! A_{e_{2}} ! \cdots A_{e_{1}+e_{2}+e_{3}} !} \quad \text { as } \quad k \rightarrow \infty,
$$

tihs is the result of step 2.

(step 3) Let us define

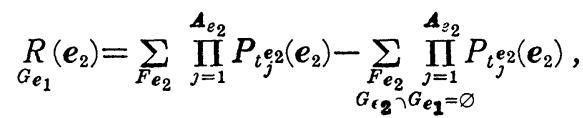

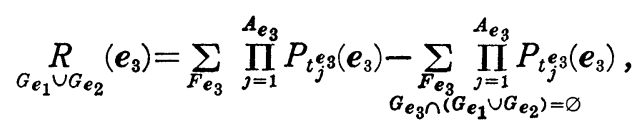

$$
\begin{aligned}
& \underset{G_{e_{1}} \cup G_{e_{2}} \cup \cdots \cup G_{e_{2}+e_{3}}}{R}\left(e_{1}+e_{2}+e_{3}\right)=\sum_{F_{1}+e_{2}+e_{3}} \prod_{j=1}^{A_{e_{1}+e_{2}+e_{3}}} P_{t_{j}^{e_{1}+e_{2}+e_{3}}\left(e_{1}+e_{2}+e_{3}\right)}
\end{aligned}
$$

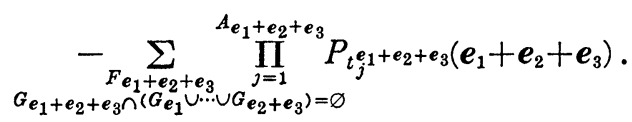

In this step, we want to prove that each of $R_{*}(\boldsymbol{i})$ in (2.9) tends to 0 as $k \rightarrow \infty$; that is,

$$
\lim _{k \rightarrow \infty} R_{*}(\boldsymbol{i})=0 \quad \text { for every } \quad \boldsymbol{i} \in \boldsymbol{E}-\left\{\mathbf{0}, \boldsymbol{e}_{1}\right\}
$$

where $*$ means the union of $G$ 's depending on $i$.

It is easy to see that for sufficient large $k$

$$
\sum_{F i} \prod_{j=1}^{n} P_{t_{j}^{i}}(i) \leqq\left[\sum_{t_{j}^{i}=1}^{n_{k}} P_{t_{j}^{i}}(i)\right]^{n} \leqq\left(\lambda_{i}+\varepsilon\right)^{n} . \quad\left(A_{i}=n\right) .
$$


It is obvious from (2.9) that $R_{*}\left(\boldsymbol{e}_{2}\right)$ is nonnegative, because the probability is nonnegative and $R_{*}\left(\boldsymbol{e}_{2}\right)$ may be estimated as follows:

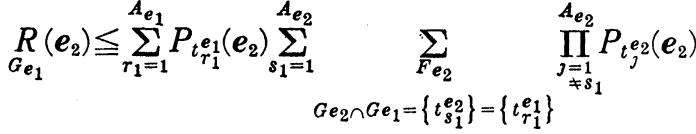

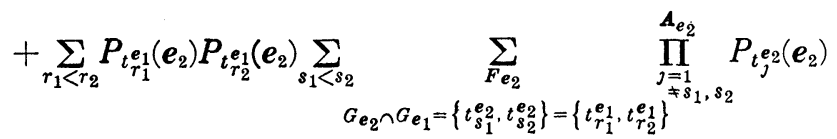

$$
\begin{aligned}
& +\cdots \\
& +\sum_{r_{1}<\cdots<r_{n}} \prod_{j=1}^{n} P_{t_{r_{j}}^{e_{1}}}\left(\boldsymbol{e}_{2}\right) \sum_{s_{1}<\cdots<s_{n}}
\end{aligned}
$$

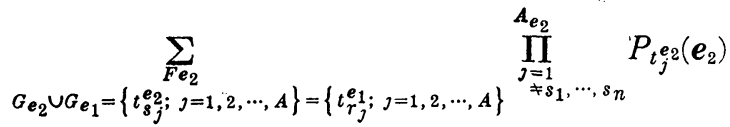

$$
\begin{aligned}
& \leqq\left(\begin{array}{c}
A_{e_{1}} \\
1
\end{array}\right)\left[1-\min _{g} P_{g}(\mathbf{0})\right]\left(\begin{array}{c}
A_{e_{2}} \\
1
\end{array}\right)\left(\lambda_{e_{2}}+\varepsilon\right)^{A_{e_{2}-1}} \\
& +\left(\begin{array}{c}
A_{e_{1}} \\
2
\end{array}\right)\left[1-\min P_{g}(\mathbf{0})\right]^{2}\left(\begin{array}{c}
A_{e_{2}} \\
2
\end{array}\right)\left(\lambda_{e_{2}}+\varepsilon\right)^{A_{e_{2}}-2} \\
& +\cdots \\
& +\left(\begin{array}{c}
A_{e_{1}} \\
A
\end{array}\right)\left[1-\min P_{g}(\mathbf{0})\right]^{A}\left(\begin{array}{c}
A_{e_{2}} \\
A
\end{array}\right)\left(\lambda_{e_{2}}+\varepsilon\right)^{A_{e_{2}-A}},
\end{aligned}
$$

By (2.4) and (2.11), the right hand side of the last inequality tends to 0 as $k \rightarrow \infty$. So we have $\underset{G e_{1}}{R}\left(\boldsymbol{e}_{2}\right) \rightarrow 0$ as $k \rightarrow \infty$. In the same way, we can proved each $R_{*}(\boldsymbol{i})$ of (2.9) tends to 0 as $k \rightarrow \infty$, for every $\boldsymbol{i} \in \boldsymbol{E}$, and we finish step 3.

Now we prove theorem 1 as follows. By the definition of $B_{n_{k}}(\boldsymbol{A})$ and (2.9), we have

$$
\begin{aligned}
& B_{n_{k}}(\boldsymbol{A})=\left\{\sum_{\boldsymbol{F}_{\boldsymbol{e}_{1}}} \prod_{j=1}^{A_{\boldsymbol{e}_{1}}} P_{t_{j}^{\boldsymbol{e}_{1}}}\left(\boldsymbol{e}_{1}\right) \sum_{\boldsymbol{F e}_{2}} \prod_{j=1}^{A_{\boldsymbol{e}_{2}}} P_{t_{j}^{\boldsymbol{e}_{2}}}\left(\boldsymbol{e}_{2}\right) \sum_{\boldsymbol{F}_{\boldsymbol{e}_{3}}} \prod_{j=1}^{A_{\boldsymbol{e}_{3}}} P_{t_{j} e_{3}\left(\boldsymbol{e}_{3}\right)}\right. \\
& \sum_{\boldsymbol{F}_{e_{1}+e_{2}}} \prod_{j=1}^{A_{e_{1}+e_{2}}} P_{t_{j}^{e_{1}+e_{2}}}\left(\boldsymbol{e}_{1}+\boldsymbol{e}_{2}\right) \sum_{\boldsymbol{F}_{\boldsymbol{e}_{1}+e_{3}}} \prod_{j=1}^{A_{e_{1}+e_{3}}} P_{t_{j}^{\boldsymbol{e}_{1}+e_{3}}}\left(\boldsymbol{e}_{1}+\boldsymbol{e}_{3}\right) \\
& \sum_{\boldsymbol{e}_{2}+\boldsymbol{e}_{3}} \prod_{j=1}^{A_{e_{2}+e_{3}}} P_{t_{j}} e_{2}+e_{3}\left(\boldsymbol{e}_{2}+\boldsymbol{e}_{3}\right) \\
& \left.\sum_{\boldsymbol{F}_{1}+e_{2}+e_{3}} \prod_{j=1}^{A_{e_{1}+e_{2}}+e_{3}} P_{t_{j}}^{e_{1}+e_{2}+e_{3}}\left(\boldsymbol{e}_{1}+\boldsymbol{e}_{2}+\boldsymbol{e}_{3}\right)\right\}
\end{aligned}
$$




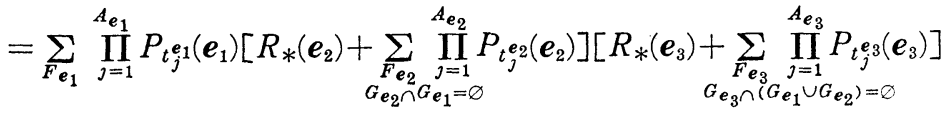

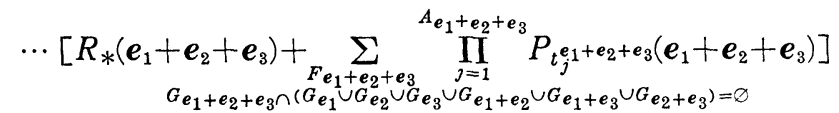

$$
\begin{aligned}
& =C_{n_{k}}(\boldsymbol{A})+F(\boldsymbol{R}) \text {, }
\end{aligned}
$$

where

$$
\boldsymbol{R}=\left(R_{*}\left(\boldsymbol{e}_{2}\right), R_{*}\left(\boldsymbol{e}_{3}\right), R_{*}\left(\boldsymbol{e}_{1}+\boldsymbol{e}_{2}\right), R_{*}\left(\boldsymbol{e}_{1}+\boldsymbol{e}_{3}\right), R_{*}\left(\boldsymbol{e}_{2}+\boldsymbol{e}_{3}\right), R_{*}\left(\boldsymbol{e}_{1}+\boldsymbol{e}_{2}+\boldsymbol{e}_{3}\right)\right),
$$

and $F$ is a polynomial of $R_{*}\left(\boldsymbol{e}_{2}\right), \cdots, R_{*}(\boldsymbol{i}), \cdots, R_{*}\left(\boldsymbol{e}_{1}+\boldsymbol{e}_{2}+\boldsymbol{e}_{3}\right)$ which coefficients may be expressed by the product of $\Sigma$ 's, and we denote $F$ by the following:

$$
\begin{aligned}
& F(\boldsymbol{R})=\sum_{F \boldsymbol{e}_{1}} \prod_{j=1}^{A_{e_{1}}} P_{t_{j}^{e_{1}}\left(\boldsymbol{e}_{1}\right) R_{*}\left(\boldsymbol{e}_{2}\right)} \sum_{F \boldsymbol{e}_{3}} \prod_{\substack{j=1 \\
G_{e_{3}} \cap\left(G_{e_{1}} \cup G_{e_{2}}\right)=\varnothing}}^{A_{e_{3}}} P_{t_{j}^{e_{3}}}\left(\boldsymbol{e}_{3}\right) \cdots
\end{aligned}
$$

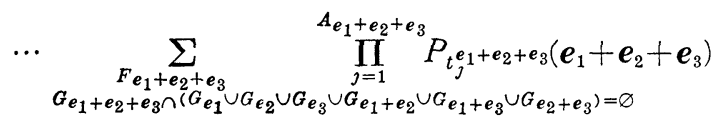

$$
\begin{aligned}
& +\sum_{F_{e_{1}}} \prod_{j=1}^{A_{e_{1}}} P_{t_{j}^{e_{1}}\left(\boldsymbol{e}_{1}\right)} \sum_{\substack{F_{\boldsymbol{e}_{2}} \\
G_{e_{2} \cap} \cap G_{e_{1}=\varnothing}}}^{A_{e_{2}}} P_{\boldsymbol{e}_{j}} P_{\boldsymbol{e}_{3}}\left(\boldsymbol{e}_{3}\right) R_{*}\left(\boldsymbol{e}_{3}\right) \cdots
\end{aligned}
$$

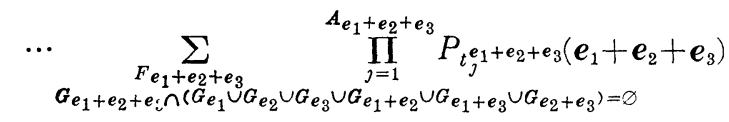

$$
\begin{aligned}
& +\cdots
\end{aligned}
$$

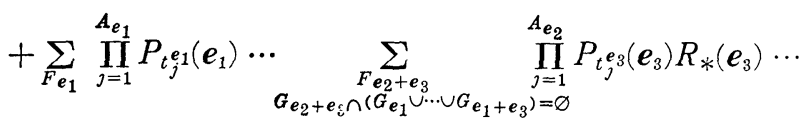

$$
\begin{aligned}
& \cdots R_{*}\left(e_{1}+e_{2}+e_{3}\right) \\
& +\cdots
\end{aligned}
$$

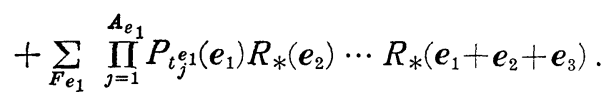

By (2.10), we get $F(\boldsymbol{R}) \rightarrow 0$, and by (2.8), (2.12) we have

$$
C_{n_{k}}(\boldsymbol{A}) \rightarrow \frac{\lambda_{e_{1}}^{A_{e_{1}}} \lambda_{e_{2}}^{A} e_{e_{2}} \cdots \lambda_{e_{1}+e_{2}+e_{3}}^{A_{e_{e_{1}}+e_{2}+e_{3}}}}{A_{e_{1}} ! A_{e_{2}} ! \cdots A_{e_{1}+e_{2}+e_{3}} !} \quad \text { as } \quad k \rightarrow \infty .
$$

It is easy to see that 


$$
C_{n_{k}}(\boldsymbol{A}) \leqq A_{n_{k}}(\boldsymbol{A}) \leqq\left(\frac{1}{\min P_{\jmath}(\mathbf{0})}\right)^{\sum_{i \in \boldsymbol{E}}^{\boldsymbol{A}_{i}}} C_{n_{k}}(\boldsymbol{A}),
$$

and by (2.4), (3.13), we have

$$
A n_{k}(\boldsymbol{A}) \rightarrow \frac{\lambda_{e_{1}}^{A_{e_{1}}} \lambda_{e_{2}}^{A_{e_{2}}} \cdots \lambda_{e_{1}+e_{2}+e_{3}}^{A_{e_{2}+e_{2}+e_{3}}}}{A_{e_{1}} ! A_{e_{2}} ! \cdots A_{e_{1}+e_{2}+e_{3}} !} \quad \text { as } \quad k \rightarrow \infty .
$$

The relations (2.6) and (2.14) finish the proof of theorem 1.

\section{Conditions necessary for Poisson approximation.}

The converse assertion of theorem 1 is also valid, but the proof is quite different. Let us show it by the following theorem.

THEOREM 2. If the condition (2.5) (for the sums of independent Bernoulli vectors which may not be identically distributed) is satisfied, then we have (2.3) and (2.4).

In order to prove theorem 2, we are going to show lemma 1 and lemma 2 .

LEMMA 1. If the condition (2.5) is satisfied, then we have

$$
\max _{1 \leq g \leqq n_{k}}\left[P_{g}(\boldsymbol{i}) / P_{\boldsymbol{g}}(\mathbf{0})\right] \rightarrow 0 \quad \text { as } k \rightarrow \infty \text {, for every } \boldsymbol{i} \in \boldsymbol{E},
$$

and

$$
\sum_{j-1}^{n_{k}} P_{j}(\boldsymbol{i}) / P_{j}(\mathbf{0}) \rightarrow \lambda_{\boldsymbol{t}} \quad \text { as } \quad k \rightarrow \infty, \text { for every } \quad \boldsymbol{i} \in \boldsymbol{E}
$$

Proof. We shall prove lemma 1 by the following four steps which can be obtained from (2.5) and using (2.2) for given $s$.

(step 1) Put $\boldsymbol{s}=\mathbf{0}$ in the relation (2.5), it is obvious to obtain

$$
\prod_{g=1}^{n_{k}} P_{g}(\mathbf{0}) \rightarrow e^{-\left(\lambda e_{1}+\lambda e_{2}+\cdots+\lambda e_{1}+e_{2}+e_{3}\right)} \quad \text { as } \quad k \rightarrow \infty .
$$

(step 2) Put $s=e_{\imath}$ and $s=2 \boldsymbol{e}_{i}$ in (2.5) and using (3.3), we obtain

$$
\sum_{t_{1}^{e_{i}=1}}^{n_{k}} P_{t_{1}^{e_{i}}}\left(\boldsymbol{e}_{2}\right) / P_{t_{1}^{e_{i}}}(\mathbf{0}) \rightarrow \lambda_{e_{\dot{\boldsymbol{e}}}}, \quad i=1,2,3 \quad \text { as } \quad k \rightarrow \infty,
$$

because the solution of $[C]$ is

$$
\left[\begin{array}{cc}
A_{\boldsymbol{e}_{\boldsymbol{i}}} & A_{\boldsymbol{\imath}}\left(\boldsymbol{i} \neq \boldsymbol{e}_{i}\right) \\
1 & 0
\end{array}\right] \text { for } \boldsymbol{s}=\boldsymbol{e}_{\imath}\left(\begin{array}{l}
\text { see example } 2 \text { which } \\
\text { explains the getting way } \\
\text { for the solution. }
\end{array}\right)
$$

(3.2.1) means (3.2) being valid for $\boldsymbol{i}=\boldsymbol{e}_{\imath}(i=1,2,3)$, and 


$$
\sum_{t_{1}^{e_{i}<t_{2}^{e_{i}}}} \prod_{j=1}^{2}\left[P_{t_{j}^{e_{i}}}\left(\boldsymbol{e}_{\imath}\right) / P_{t_{j}^{e_{i}}}(\mathbf{0})\right] \rightarrow \lambda_{e_{i}}^{2} / 2 \quad \text { as } \quad k \rightarrow \infty,
$$

because the solution of $[C]$ is

$$
\left[\begin{array}{cl}
A_{e_{i}} & A_{\boldsymbol{u}}\left(\boldsymbol{i} \neq \boldsymbol{e}_{\imath}\right) \\
2 & 0
\end{array}\right] \quad \text { for } \quad \boldsymbol{s}=2 \boldsymbol{e}_{\imath}
$$

By (3.2.1) and (3.4), we get

$$
\sum_{t_{1}^{e_{i}=1}}^{n_{k}}\left[P_{t_{1}^{e_{i}}}\left(\boldsymbol{e}_{2}\right) / P_{t_{1}^{e_{i}}}(\mathbf{0})\right]^{2} \rightarrow 0 \quad \text { as } k \rightarrow \infty,
$$

which implies that

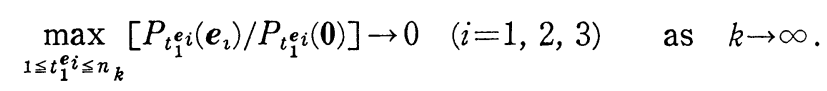

(3.1.1) means (3.1) being valid for $\boldsymbol{i}=\boldsymbol{e}_{\imath}(i=1,2,3)$.

(step 3) Put $\boldsymbol{s}=\boldsymbol{e}_{i}+\boldsymbol{e}_{\jmath}(1 \leqq i<j \leqq 3)$ in (2.5), we obtain

$$
\begin{aligned}
& \sum_{t_{1}^{e_{i}+e_{j=1}} n_{k}}^{n_{k}} P_{t_{1}^{e_{i}+\boldsymbol{e}_{j}}}\left(\boldsymbol{e}_{i}+\boldsymbol{e}_{j}\right) / P_{t_{1}^{e_{i}+e_{j}}}(\mathbf{0}) \\
& \quad+\sum_{t_{1}^{e_{i}=1}}^{n_{k}} P_{t_{1}^{e_{i}}}\left(\boldsymbol{e}_{\imath}\right) / P_{t_{1}^{e_{i}}}(\mathbf{0}) \sum_{\substack{\sum_{1}^{j_{j}} \\
\neq t_{1}^{i}}}^{n_{k}} P_{t_{1}^{e_{j}}}\left(\boldsymbol{e}_{j}\right) / P_{t_{1}^{e_{j}}}(0) \\
& \rightarrow \lambda_{e_{i}+\boldsymbol{e}_{j}}+\lambda_{\boldsymbol{e}_{i}} \cdot \lambda_{e_{j}},
\end{aligned}
$$

because the solution of $[C]$ is

$$
\left[\begin{array}{cccc}
A_{\boldsymbol{e}_{i}} & A_{\boldsymbol{e}_{j}} & A_{\boldsymbol{e}_{i}+\boldsymbol{e}_{\jmath}} & A_{\boldsymbol{e}}\left(\boldsymbol{i} \neq \boldsymbol{e}_{\imath}, \boldsymbol{e}_{\jmath}, \boldsymbol{e}_{i}+\boldsymbol{e}_{\jmath}\right) \\
1 & 1 & 0 & 0 \\
0 & 0 & 1 & 0
\end{array}\right] \text { for } \quad \boldsymbol{s}=\boldsymbol{e}_{i}+\boldsymbol{e}_{\jmath} \quad(1 \leqq \imath<\jmath \leqq 3)
$$

Since

$$
\begin{aligned}
& \sum_{t_{1}^{e_{i}} n_{k}}^{n_{k}} P_{t_{1}^{e_{i}}}\left(\boldsymbol{e}_{\imath}\right) / P_{t_{1}^{e_{i}}}(\mathbf{0}) \cdot P_{t_{1}^{e_{i}}}\left(\boldsymbol{e}_{j}\right) / P_{t_{1}^{e_{i}}}(\mathbf{0}) \\
& \leqq\left(\lambda_{e_{i}}+\boldsymbol{\varepsilon}\right) \cdot \max _{t_{1}^{e_{i}}}\left[P_{t_{1}^{e_{i}}}\left(\boldsymbol{e}_{j}\right) / P_{t_{1}^{e_{i}}}(\mathbf{0})\right] \\
& \rightarrow 0 \quad \text { (by (3.1.1), (3.2.1)), }
\end{aligned}
$$

then by (3.2.1), we can obtain the second term of the left side of (3.5) tends to $\lambda_{e_{i}} \cdot \lambda_{e_{j}}$, and by (3.5), we have

$$
\sum_{t_{1}^{e_{i}+e_{j=1}}}^{n_{k}} P_{t_{1}^{e_{i}+e_{j}}}\left(\boldsymbol{e}_{i}+\boldsymbol{e}_{j}\right) / P_{t_{1}^{e_{i}+e_{j}}}(\mathbf{0}) \rightarrow \lambda_{\boldsymbol{e}_{i}+e_{j}} \quad(1 \leqq i<\jmath \leqq 3) \quad \text { as } \quad k \rightarrow \infty
$$

(3.2.2) means (3.2) being valid for $\boldsymbol{i}=\boldsymbol{e}_{i}+\boldsymbol{e}_{\boldsymbol{\jmath}}(\boldsymbol{i}=1,2,3)$. Similarly put $\boldsymbol{s}=2\left(\boldsymbol{e}_{i}+\boldsymbol{e}_{\jmath}\right)$ $(1 \leqq i<j \leqq 3)$ in the relation $(2.5)$, we get 


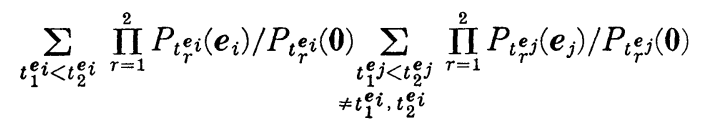

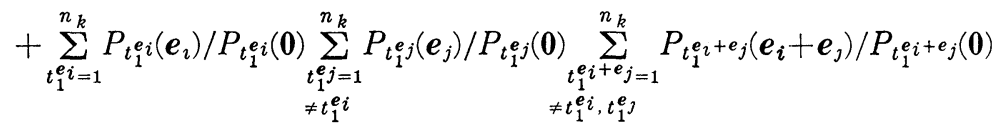

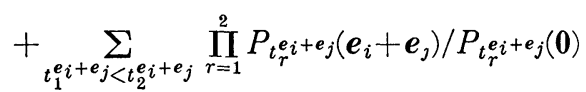

$$
\begin{aligned}
& \rightarrow\left[\left(\lambda_{e_{i}}\right)^{2} / 2\right] \cdot\left[\left(\lambda_{e_{j}}\right)^{2} / 2\right]+\lambda_{e_{i}} \cdot \lambda_{e_{j}} \cdot \lambda_{e_{i}+e_{j}}+\left(\lambda_{e_{i}+e_{i}}\right)^{2} / 2,
\end{aligned}
$$

because the solution of $[C]$ is

$$
\left[\begin{array}{cccl}
A \boldsymbol{e}_{\boldsymbol{\imath}} & A \boldsymbol{e}_{\boldsymbol{j}} & A \boldsymbol{e}_{i}+\boldsymbol{e}_{\boldsymbol{j}} & A_{\boldsymbol{t}}\left(\boldsymbol{i} \neq \boldsymbol{e}_{\imath}, \boldsymbol{e}_{j}, \boldsymbol{e}_{i}+\boldsymbol{e}_{j}\right) \\
2 & 2 & 0 & 0 \\
1 & 1 & 1 & 0 \\
0 & 0 & 2 & 0
\end{array}\right] \text { for } \boldsymbol{s}=2\left(\boldsymbol{e}_{i}+\boldsymbol{e}_{j}\right) \quad(1 \leqq i<j \leqq 3)
$$

Let us consider the first term of the left side of (3.6). By (3.1.1), (3.2.1) and having the similar consideration deriving (2.10), we can obtain

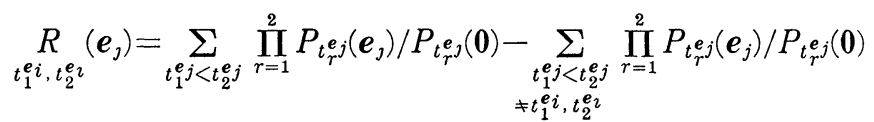

$$
\begin{aligned}
& \leqq P_{t_{1}^{e_{i}}}\left(\boldsymbol{e}_{j}\right) / P_{t_{1}^{e_{i}}}(\mathbf{0}) \sum_{t_{1}^{e_{i}<t_{2}^{\boldsymbol{e}_{j}}}} P_{t_{2}^{e_{j}}}\left(\boldsymbol{e}_{j}\right) / P_{t_{2}^{e_{j}} j}(\mathbf{0}) \\
& +P_{t_{2}^{e_{i}}}\left(\boldsymbol{e}_{j}\right) / P_{t_{2}^{e_{i}}}(\mathbf{0}) \sum_{t_{2}^{e_{i}<t_{2}^{\boldsymbol{e}_{j}}}} P_{t_{2}^{e_{j}}}\left(\boldsymbol{e}_{j}\right) / P_{t_{2}^{e_{j}}}(\mathbf{0}) \\
& +P_{t_{1}^{e_{i}}}\left(\boldsymbol{e}_{j}\right) / P_{t_{1}^{e_{i}}}(\mathbf{0}) \sum_{t_{1}^{e_{j}<t_{1}^{e_{i}}}} P_{t_{1}^{e_{j}}}\left(\boldsymbol{e}_{j}\right) / P_{t_{1}^{e_{j}}}(\mathbf{0}) \\
& +P_{t_{2}^{e_{i}}\left(\boldsymbol{e}_{j}\right) / P_{t_{2}^{e_{i}}}(\mathbf{0})} \sum_{t_{1}^{e_{j}<t_{2}^{e_{i}}}} P_{t_{1}^{e_{j}}}\left(\boldsymbol{e}_{j}\right) / P_{t_{1}^{e_{j}}}(\mathbf{0}) \\
& +P_{t_{1}^{e_{i}}\left(\boldsymbol{e}_{j}\right)} / P_{t_{1}^{e_{i}}(\mathbf{0})} \cdot P_{t_{2}^{e_{i}}}\left(\boldsymbol{e}_{j}\right) / P_{t_{2}^{e_{i}}(\mathbf{0})} \\
& \leqq \max _{g}\left[P_{g}\left(\boldsymbol{e}_{j}\right) / P_{g}(\boldsymbol{0})\right] \cdot 4\left(\lambda \boldsymbol{e}_{j}+\boldsymbol{\varepsilon}\right) \\
& +\max \left[P_{g}\left(\boldsymbol{e}_{j}\right) / P_{g}(\mathbf{0})\right] \cdot \max \left[P_{\boldsymbol{g}}\left(\boldsymbol{e}_{j}\right) / P_{\boldsymbol{g}}(\mathbf{0})\right] \\
& \rightarrow 0 \quad \text { (by (3.1.1), (3.2.1)), }
\end{aligned}
$$

and by (3.4) we have

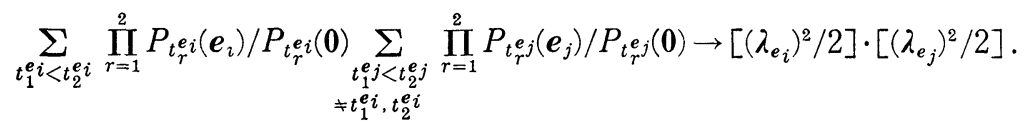

The second term of the left side of (3.6) may be represented by 


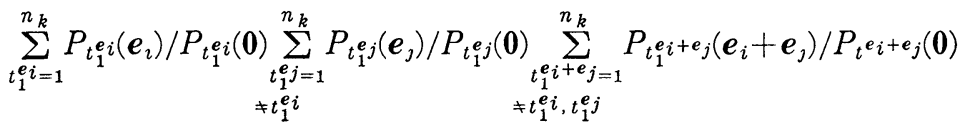

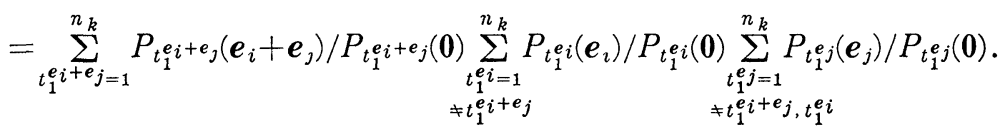

By (3.1.1), (3.2.1) and having the similar consideration deriving (2.10), we can obtain

$$
\begin{aligned}
\underset{t_{1}^{e_{i}+e_{j}}}{R\left(\boldsymbol{e}_{\imath}\right)} & =\sum_{t_{1}^{e_{i}=1}}^{n_{k}} P_{t_{1}^{e_{i}}}\left(\boldsymbol{e}_{\imath}\right) / P_{t_{1}^{e_{i}}}(\mathbf{0})-\sum_{\substack{t_{1}^{e_{i}=1} \\
\neq t_{1}^{e_{i}+e_{j}}}}^{n_{k}} P_{t_{1}^{e_{i}}}\left(\boldsymbol{e}_{\imath}\right) / P_{t_{1}^{e_{i}}}(\mathbf{0}) \\
& =P_{t_{1}^{e_{i}}+\boldsymbol{e}_{j}}\left(\boldsymbol{e}_{\imath}\right) / P_{t_{1}^{e_{i}}+e_{j}}(\mathbf{0}) \\
& \leqq \max _{\boldsymbol{g}}\left[P_{g}\left(\boldsymbol{e}_{\imath}\right) / P_{g}(\mathbf{0})\right] \\
& \rightarrow 0, \quad(\text { by }(3.1 .1))
\end{aligned}
$$

and

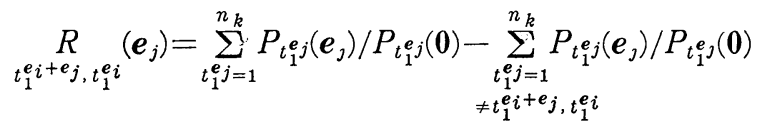

$$
\begin{aligned}
& =P_{t_{1}^{e_{i}}+e_{j}}\left(\boldsymbol{e}_{j}\right) / P_{t^{e_{i}+e_{j}}}(\mathbf{0})+P_{t_{1}^{e_{i}}}\left(\boldsymbol{e}_{j}\right) / P_{t_{1}^{e_{i}}}(\mathbf{0}) \\
& \leqq 2 \max _{g}\left[P_{g}\left(\boldsymbol{e}_{\imath}\right) / P_{g}(\mathbf{0})\right] \\
& \rightarrow 0, \quad(\text { by }(3.1 .1))
\end{aligned}
$$

and by (3.2.1), (3.2.2), we have

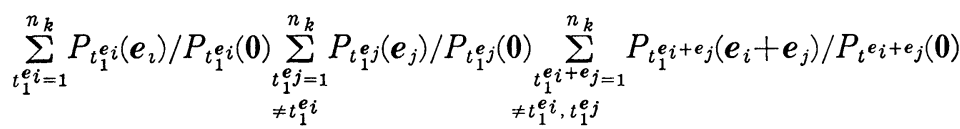

$$
\begin{aligned}
& \rightarrow \lambda_{e_{i}} \cdot \lambda_{e_{j}} \cdot \lambda_{e_{i}+e_{j}} \cdot
\end{aligned}
$$

From the discussion above and by (3.6), we have

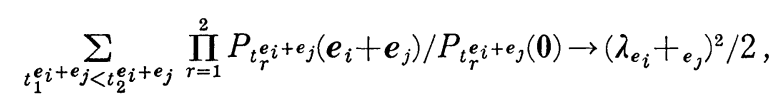

and by (3.2.2), (3.7), we get

$$
\sum_{t_{1}^{e_{i}+\boldsymbol{e}_{j=1}}}^{n_{k}}\left[P_{t_{1}^{e_{i}}+\boldsymbol{e}_{j}}\left(\boldsymbol{e}_{i}+\boldsymbol{e}_{j}\right) / P_{t_{1}^{e_{i}+\boldsymbol{e}_{j}}}(\mathbf{0})\right]^{2} \rightarrow 0
$$

which implies that 


$$
\max _{\boldsymbol{t}_{1}^{\boldsymbol{e}_{i}+\boldsymbol{e}_{j}}}\left[P_{t_{1}^{e_{i}+\boldsymbol{e}_{\jmath}}}\left(\boldsymbol{e}_{i}+\boldsymbol{e}_{j}\right) / P_{t_{1}^{e_{i}+\boldsymbol{e}_{\jmath}}}(\boldsymbol{0})\right] \rightarrow 0, \quad(1 \leqq \imath<\jmath \leqq 3), \text { as } \quad k \rightarrow \infty .
$$

(3.1.2) means (3.1) being valid for $\boldsymbol{i}=\boldsymbol{e}_{i}+\boldsymbol{e}_{\jmath}(1 \leqq \imath<\jmath \leqq 3)$.

The solution of $[C]$ for fixed $s$ will be given in the following example.

Example 2. For $\boldsymbol{s}=2\left(\boldsymbol{e}_{1}+\boldsymbol{e}_{2}\right)=(2,2,0)$ we have

$$
\begin{aligned}
& A_{100}+A_{101}+A_{110}+A_{111}=2 \\
& A_{010}+A_{011}+A_{110}+A_{111}=2 \\
& A_{001}+A_{011}+A_{101}+A_{111}=0
\end{aligned}
$$

The solution of $[C]$ is given by the table.

$$
\left[\begin{array}{cccc}
A_{100} & A_{010} & A_{110} & A_{\boldsymbol{\varepsilon}}\left(\boldsymbol{i} \neq \boldsymbol{e}_{1}, \boldsymbol{e}_{2}, \boldsymbol{e}_{1}+\boldsymbol{e}_{2}\right) \\
2 & 2 & 0 & 0 \\
1 & 1 & 1 & 0 \\
0 & 0 & 2 & 0
\end{array}\right]
$$

(step 4) In the same way as step 3 , put $\boldsymbol{s}=\boldsymbol{e}_{1}+\boldsymbol{e}_{2}+\boldsymbol{e}_{3}$ in (2.5) we obtain

$$
\begin{aligned}
& \sum_{t_{1}^{e_{1}+e_{2}+e_{3}=1}}^{n_{k}} P_{t_{1}^{e_{1}+e_{2}+e_{3}}}\left(\boldsymbol{e}_{1}+\boldsymbol{e}_{2}+\boldsymbol{e}_{3}\right) / P_{t_{1}^{e_{1}+e_{2}+e_{3}}}(\boldsymbol{0}) \\
& +\sum_{t_{1}^{e_{1}=1}}^{n_{k}} P_{t_{1}^{e_{1}}}\left(\boldsymbol{e}_{1}\right) / P_{t_{1}^{e_{1}}(\mathbf{0})} \sum_{\substack{t_{1}^{e_{2}+e_{3}} \\
\neq t_{1}^{e_{1}}}}^{n_{k}} P_{t_{1}^{e_{2}}+e_{3}}\left(\boldsymbol{e}_{2}+\boldsymbol{e}_{3}\right) / P_{t_{1}^{e_{2}}+e_{3}}(\mathbf{0}) \\
& +\sum_{t_{1}^{e_{2}=1}}^{n_{k}} P_{t_{1}^{e_{2}}}\left(\boldsymbol{e}_{2}\right) / P_{t_{1}^{e_{2}}(\mathbf{0})} \sum_{\substack{t_{1}^{e_{1}+e_{3}} \\
\neq t_{1}^{e_{2}}}}^{n_{k}} P_{t_{1}^{e_{1}+e_{3}}}\left(\boldsymbol{e}_{1}+\boldsymbol{e}_{3}\right) / P_{t_{1}^{e_{1}}+\boldsymbol{e}_{3}}(\mathbf{0})
\end{aligned}
$$

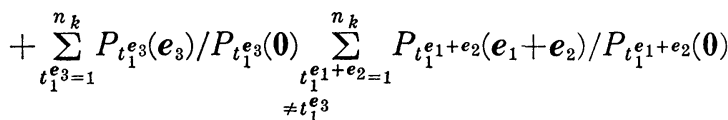

$$
\begin{aligned}
& +\sum_{t_{1}^{e_{1}=1}}^{n_{k}} P_{t_{1}^{e_{1}}}\left(\boldsymbol{e}_{1}\right) / P_{t_{1}^{e_{1}}}(\boldsymbol{0}) \sum_{\substack{t_{1}^{e_{2}=1} \\
\neq t_{1}^{e_{1}}}}^{n_{k}} P_{t_{1}^{e_{2}}}\left(\boldsymbol{e}_{2}\right) / P_{t_{1}^{e_{2}}}(\mathbf{0}) \sum_{\substack{t_{1}^{e_{3}} \\
\neq=1 \\
t_{1}^{e_{1}}, t_{1}^{e_{2}}}}^{n_{k}} P_{t_{1}^{e_{3}}}\left(\boldsymbol{e}_{3}\right) / P_{t_{1}^{e_{3}}}(\mathbf{0}) \\
& \rightarrow \lambda_{e_{1}+e_{2}+e_{3}}+\lambda_{e_{1}} \cdot \lambda_{e_{2}+e_{3}}+\lambda_{e_{2}} \cdot \lambda_{e_{1}+e_{3}}+\lambda_{e_{3}} \cdot \lambda_{e_{1}+e_{2}}+\lambda_{e_{1}} \cdot \lambda_{e_{2}} \cdot \lambda_{e_{3}},
\end{aligned}
$$

because the solution of $[C]$ is 


$$
\left[\begin{array}{ccccccc}
A_{e_{1}} & A_{e_{2}} & A_{e_{3}} & A_{e_{1}+e_{2}} & A_{e_{1}+e_{3}} & A_{e_{2}+e_{3}} & A_{e_{1}+e_{2}+e_{3}} \\
0 & 0 & 0 & 0 & 0 & 0 & 1 \\
1 & 0 & 0 & 0 & 0 & 1 & 0 \\
0 & 1 & 0 & 0 & 1 & 0 & 0 \\
0 & 0 & 1 & 1 & 0 & 0 & 0 \\
1 & 1 & 1 & 0 & 0 & 0 & 0
\end{array}\right], \text { for } s=\boldsymbol{e}_{1}+\boldsymbol{e}_{2}+\boldsymbol{e}_{3}
$$

Since

$$
\begin{aligned}
& \sum_{t_{1}^{e_{1}=1}}^{n_{k}} P_{t_{1}^{e_{1}}}\left(\boldsymbol{e}_{1}\right) / P_{t_{1}^{e_{1}}}(\mathbf{0}) \cdot P_{t_{1}^{e_{1}}}\left(\boldsymbol{e}_{2}+\boldsymbol{e}_{3}\right) / P_{t_{1}^{e_{1}}}(\mathbf{0}) \\
& \leqq \max _{\boldsymbol{g}}\left[P_{\boldsymbol{g}}\left(\boldsymbol{e}_{2}+\boldsymbol{e}_{3}\right) / P_{g}(\mathbf{0})\right] \sum_{t_{1}^{e_{1}=1}}^{n_{k}} P_{t_{1}^{e_{1}}}\left(\boldsymbol{e}_{1}\right) / P_{t_{1}^{e_{1}}}(\mathbf{0}) \\
& \rightarrow 0, \quad(\text { by }(3.1 .2),(3.2 .1)),
\end{aligned}
$$

and similarly we have

$$
\begin{aligned}
& \sum_{t_{1}^{e_{2}=1}}^{n_{k}} P_{t_{1}^{e_{2}}}\left(\boldsymbol{e}_{2}\right) / P_{t_{1}^{e_{2}}}(\mathbf{0}) \cdot P_{t_{1}^{e_{2}}}\left(\boldsymbol{e}_{1}+\boldsymbol{e}_{3}\right) / P_{t_{1}^{e_{2}}}(\mathbf{0}) \\
& \leqq \max _{\boldsymbol{g}}\left[P_{g}\left(\boldsymbol{e}_{1}+\boldsymbol{e}_{3}\right) / P_{g}(\mathbf{0})\right] \sum_{t_{1}^{e_{2}=1}}^{n_{k}} P_{t_{1}^{e_{1}}\left(\boldsymbol{e}_{2}\right) / P_{t_{1}^{e_{2}}}(\mathbf{0})} \\
& \rightarrow 0, \quad(\text { by }(3.1 .2),(3.2 .1)),
\end{aligned}
$$

and

$$
\begin{aligned}
& \sum_{t_{1}^{e_{3}=1}}^{n_{k}} P_{t_{1}^{e_{3}}}\left(\boldsymbol{e}_{3}\right) / P_{t_{1}^{e_{3}}}(\mathbf{0}) \cdot P_{t_{1}^{e_{3}}}\left(\boldsymbol{e}_{1}+\boldsymbol{e}_{2}\right) / P_{t_{1}^{e_{3}}}(\mathbf{0}) \\
& \leqq \max _{g}\left[P_{g}\left(\boldsymbol{e}_{1}+\boldsymbol{e}_{2}\right) / P_{g}(\mathbf{0})\right] \sum_{t_{1}^{e_{3}} n_{k}}^{n_{k}} P_{t_{1}^{e_{3}}}\left(\boldsymbol{e}_{3}\right) / P_{t_{1}^{e_{3}}}(\mathbf{0}) \\
& \rightarrow 0, \quad(\text { by }(3.1 .2),(3.2 .1)),
\end{aligned}
$$

and

$$
\begin{aligned}
& \sum_{t_{1}^{e_{1}=1}}^{n_{k}} P_{t_{1}^{e_{1}}}\left(\boldsymbol{e}_{1}\right) / P_{t_{1}^{e_{1}}}(\mathbf{0}) \cdot P_{t_{1}^{e_{1}}\left(\boldsymbol{e}_{2}\right) / P_{t_{1}^{e_{1}}}(\mathbf{0})} \sum_{t_{1}^{e_{3}=1}}^{n_{k}} P_{t_{1}^{e_{3}}}\left(\boldsymbol{e}_{3}\right) / P_{t_{1}^{e_{3}}}(\mathbf{0}) \\
& +\sum_{t_{1}^{e_{1}=1}}^{n_{k}} P_{t_{1}^{e_{1}}}\left(\boldsymbol{e}_{1}\right) / P_{t_{1}^{e_{1}}}(\mathbf{0}) \sum_{\substack{t_{1}^{t_{2}=1} \\
\neq t_{1}^{1}}}^{n_{k}} P_{t_{1}^{e_{2}}}\left(\boldsymbol{e}_{2}\right) / P_{t_{1}^{e_{2}}}(\mathbf{0}) \cdot P_{t_{1}^{e_{1}}}\left(\boldsymbol{e}_{3}\right) / P_{t_{1}^{e_{1}}}(\mathbf{0}) \\
& +\sum_{t_{1}^{e_{1}=1}}^{n_{k}} P_{t_{1}^{e_{1}}}\left(\boldsymbol{e}_{1}\right) / P_{t_{1}^{e_{1}}}(\mathbf{0}) \sum_{\substack{t_{1}^{e_{2}=1} \\
\neq t_{1}^{e_{1}}}}^{n_{k}} P_{t_{1}^{e_{2}}}\left(\boldsymbol{e}_{2}\right) / P_{t_{1}^{e_{2}}}(\mathbf{0}) \cdot P_{t_{1}^{e_{2}}}\left(\boldsymbol{e}_{3}\right) / P_{t_{1}^{e_{2}}}(\mathbf{0}) \\
& \leqq\left(\lambda_{e_{1}}+\varepsilon\right) \cdot \max _{\boldsymbol{g}}\left[P_{g}\left(\boldsymbol{e}_{2}\right) / P_{g}(\mathbf{0})\right] \cdot\left(\lambda_{e_{3}}+\varepsilon\right) \\
& +\left(\lambda_{e_{1}}+\varepsilon\right) \cdot\left(\lambda_{e_{2}}+\varepsilon\right) \cdot \max _{g}\left[P_{g}\left(\boldsymbol{e}_{3}\right) / P_{g}(\mathbf{0})\right]
\end{aligned}
$$




$$
\begin{aligned}
& +\left(\lambda_{e_{1}}+\varepsilon\right) \cdot\left(\lambda_{e_{2}}+\varepsilon\right) \cdot \max _{\boldsymbol{g}}\left[P_{g}\left(\boldsymbol{e}_{3}\right) / P_{g}(\mathbf{0})\right] \\
\rightarrow & 0, \quad(\text { by }(3.1 .1),(3.2 .1)),
\end{aligned}
$$

then by (3.2.1) and (3.2.2) we can obtain the last four terms of the left side of (3.8) tend to $\left(\lambda_{e_{1}} \cdot \lambda_{e_{2}+e_{3}}\right)+\left(\lambda_{e_{2}} \cdot \lambda_{e_{1}+e_{3}}\right)+\left(\lambda_{e_{3}} \cdot \lambda_{e_{1}+e_{2}}\right)+\left(\lambda_{e_{1}} \cdot \lambda_{e_{2}} \cdot \lambda_{e_{3}}\right)$ and by (3.8) we obtain the first term of the left side of (3.8) tend to $\lambda \boldsymbol{e}_{1}+\boldsymbol{e}_{2}+\boldsymbol{e}_{3}$; that is,

$$
\sum_{t_{1}^{e_{1}+e_{2}+e_{3=1}}}^{n_{k}} P_{t_{1}^{e_{1}}+e_{2}+e_{3}}\left(\boldsymbol{e}_{1}+\boldsymbol{e}_{2}+\boldsymbol{e}_{3}\right) / P_{t_{1}^{e_{1}+e_{2}+e_{3}}}(\boldsymbol{0}) \rightarrow \lambda_{e_{1}+e_{2}+e_{3}} .
$$

(3.2.3) means (3.2) being valid for $\boldsymbol{i}=\boldsymbol{e}_{1}+\boldsymbol{e}_{2}+\boldsymbol{e}_{3}$. Similarly, put $\boldsymbol{s}=2\left(\boldsymbol{e}_{1}+\boldsymbol{e}_{2}+\boldsymbol{e}_{3}\right)$ and having the same considering of step 3 , we get

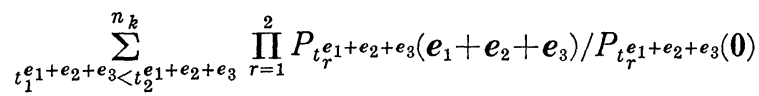

$$
\begin{aligned}
& \rightarrow\left(\lambda_{e_{1}+e_{2}+e_{3}}\right)^{2} / 2 \text {, }
\end{aligned}
$$

because the solution of $[C]$ is

$\begin{array}{cccccccc}A_{e_{1}} & A_{e_{2}} & A_{e_{3}} & A_{e_{1}+e_{2}} & A_{e_{1}+e_{3}} & A_{e_{2}+e_{3}} & A_{e_{1}+e_{2}+e_{3}} & \\ 0 & 0 & 0 & 0 & 0 & 0 & 2 & \\ 1 & 1 & 1 & 0 & 0 & 0 & 1 & \\ 1 & 0 & 0 & 0 & 0 & 1 & 1 & \\ 0 & 0 & 1 & 1 & 0 & 0 & 1 & \\ 0 & 1 & 0 & 0 & 1 & 0 & 1 & \\ 2 & 0 & 0 & 0 & 0 & 2 & 0 & \\ 2 & 1 & 1 & 0 & 0 & 1 & 0 & \\ 2 & 2 & 2 & 0 & 0 & 0 & 0 & \\ 0 & 0 & 2 & 2 & 0 & 0 & 0 & \\ 0 & 2 & 0 & 0 & 2 & 0 & 0 & \\ 0 & 0 & 0 & 1 & 1 & 1 & 0 & \\ 0 & 1 & 1 & 1 & 1 & 0 & 0 & \\ 1 & 1 & 0 & 0 & 1 & 1 & 0 & \\ 1 & 2 & 1 & 0 & 1 & 0 & 0 & \\ 1 & 0 & 1 & 1 & 0 & 1 & 0 & \\ 1 & 1 & 2 & 1 & 0 & 0 & 0, \quad \text { for } s=2\left(\boldsymbol{e}_{1}+\boldsymbol{e}_{2}+\boldsymbol{e}_{3}\right),\end{array}$

and by (3.2.3), (3.9), we can obtain

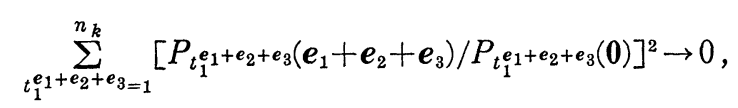

which implies that

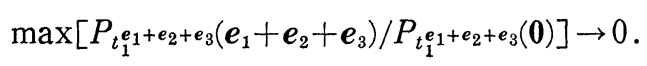

(3.1.3) means (3.1) being valid for $\boldsymbol{i}=\boldsymbol{e}_{1}+\boldsymbol{e}_{2}+\boldsymbol{e}_{3}$. The conclusions of our four 
steps finish lemma 1.

LEMMA 2. If the conditions (3.1) and (3.2) are satısfied, then we have (2.3) and (2.4).

Proof. Because $\max _{\boldsymbol{g}}\left[P_{g}(\boldsymbol{i}) / P_{\boldsymbol{g}}(\mathbf{0})\right] \rightarrow 0$, for all $\boldsymbol{i} \in \boldsymbol{E}$, we have for all $\boldsymbol{\varepsilon}>0$,

$$
\begin{aligned}
\left(2^{3}-1\right) \cdot \varepsilon & \geqq \sum_{i \in E} \max _{g}\left[P_{g}(\boldsymbol{i}) / P_{g}(\mathbf{0})\right] \\
& \geqq \max _{g} \sum_{i \in \boldsymbol{E}}\left[P_{g}(\boldsymbol{i}) / P_{g}(\mathbf{0})\right] \\
& =\max _{g}\left\{\left[1-P_{g}(\mathbf{0})\right] / P_{g}(\mathbf{0})\right\} \\
& =\max _{g}\left\{\frac{1}{P_{g}(\mathbf{0})}-1\right\} \\
& =\frac{1}{\min _{g} P_{g}(\mathbf{0})}-1 \\
& \geqq 0 \quad\left(\text { where } 1 \leqq g \leqq n_{k}\right),
\end{aligned}
$$

and we can prove

$$
\min _{1 \leqq g \leqq n_{k}} P_{g}(\mathbf{0}) \rightarrow 1 \quad \text { as } \quad k \rightarrow \infty
$$

Since

$$
\begin{aligned}
& \min _{\boldsymbol{g}} P_{g}(\mathbf{0}) \cdot \sum_{j=1}^{n_{k}} P_{j}(\boldsymbol{i}) / P_{j}(\mathbf{0}) \\
& \leqq \sum_{j=1}^{n_{k}} P_{j}(\boldsymbol{i}) \\
& \leqq \sum_{j=1}^{n_{k}} P_{j}(\boldsymbol{i}) / P_{j}(\mathbf{0})
\end{aligned}
$$

and by (3.2), (2.4), we can obtain

$$
\sum_{j=1}^{n_{k}} P_{j}(\boldsymbol{i}) \rightarrow \lambda_{\boldsymbol{i}} \quad \text { as } \quad k \rightarrow \infty, \quad \text { for every } \quad \boldsymbol{i} \in \boldsymbol{E},
$$

and we finish the proof lemma 2. The conclusions of lemma 1 and lemma 2 complete the proof of theorem 2 .

\section{Conclusion.}

In this paper, we have derived the necessary and sufficient conditions of Poisson approximation for sums of independent trivariate Bernoulli vectors which may not be identically distributed. The author considers that he has already extended the trivariate case to multivariate case, however, a little 
problem lies with the way of expressing the general notations and its refinement and hopes to report it in the near future.

\section{Acknowledgement.}

The author expresses his hearty thanks to Professor K. Kawamura for his kind and continuous guidance during the progress of this work. Not only that, Prof. K. Kawamura helps the author understand Japanese texts, papers and spends much of his valuable time to look after personal problems as well. The author knows no word to express his gratitude to him.

\section{REFERENCES}

[1] M. POLAK, Poisson approximation for sums of independent bivariate Bernoulli vectors, Kodai Math. J., 5 (1982), 408-415.

[2] K. Kawamura, The structure of trivariate Poisson distribution, Kodai Math. Sem. Rep., 28 (1976), 1-8.

Department of Mathematics

TOKyo Institute of TECHNOLOGY

Ohokayama, Meguro-kU

TOKYO 